\title{
Sofrimento psíquico em adolescentes associado ao alcoolismo familiar: possíveis fatores de risco*
}

\section{Psychological distress in adolescents associated with family alcoholism: possible risk factors*}

Vagna Cristina Leite da Silva Pereira ${ }^{1}$, Fábio Alencar de Andrade ${ }^{2}$, Lawrencita Limeira Espínola ${ }^{3}$, Elisangela Braga de Azevedo ${ }^{4}$, Jordana de Almeida Nogueira ${ }^{5}$, Maria de Oliveira Ferreira Filha ${ }^{6}$

\footnotetext{
* Este artigo é um recorte da dissertação de Mestrado defendida em 2012, junto ao Programa do Pós-Graduação em Enfermagem da Universidade Federal da Paraíba, intitulada Prevalência do Sofrimento Mental em Adolescentes que Convivem com Familiares Alcoolistas".

${ }^{1}$ Enfermeira, Mestre em Enfermagem. Discente do Programa de Pós-Graduação em Enfermagem (PPGEnf) da Universidade Federal da Paraíba (UFPB), nível Doutorado. João Pessoa, PB, Brasil. E-mail: vagna.cristina@bol.com.br.

${ }^{2}$ Estatístico, Mestre em Modelo de Decisão em Saúde. Estatístico da Secretaria de Saúde do Estado da Paraíba. João Pessoa, PB, Brasil. E-mail: fabio_estatis-

tic@yahoo.com.br.

${ }^{3}$ Enfermeira, Mestre em Enfermagem. Psicóloga da UFPB. João Pessoa, PB, Brasil. E-mail: lawrencita_@hotmail.com.

${ }^{4}$ Enfermeira, Doutora em Enfermagem. Docente da Faculdade de Ciências Médicas de Campina Grande. Campina Grande, PB, Brasil. E-mail: elisaaz@terra.com.br.

${ }^{5}$ Enfermeira, Doutora em Enfermagem. Professor Associado do PPGEnf/UFPB. João Pessoa, PB, Brasil. E-mail: jal_nogueira@yahoo.com.br.

${ }^{6}$ Enfermeira, Doutora em Enfermagem. Professor Associado do PPGEnf/UFPB. João Pessoa, PB, Brasil. E-mail: marfilha@yahoo.com.br.
}

\section{RESUMO}

Objetivou-se identificar fatores de risco preditores para sofrimento psíquico em adolescentes que convivem ou não com familiares alcoolistas. Estudo epidemiológico que selecionou 211 adolescentes em sofrimento psíquico a partir de uma amostra de 715 estudantes da rede estadual de ensino de João Pessoa/PB- Brasil. Os dados foram coletados utilizando-se três instrumentos: CAGE-familiar; Self-Reporting Questionnaire e um questionário estruturado. Para a análise, empregou-se o modelo de regressão logística. Evidenciou-se que adolescentes que conviviam com pais alcoolistas, cujas mães apresentavam maior nível de escolaridade, tiveram menor chance de manifestarem adoecimento psíquico $(O R=0,63)$. Entre os adolescentes que não conviviam com familiares alcoolistas, a maior chance para manifestação do agravo esteve associada ao nível de escolaridade paterna $(O R=1,2)$, ser do sexo feminino $(O R=1,8)$ e pertencer à família de modelo não nuclear. O planejamento de ações/intervenções que favoreçam a construção efetiva de cuidados a esses indivíduos deve considerar as singularidades socioculturais do contexto familiar.

Descritores: Enfermagem Psiquiátrica; Adolescente; Fatores de Risco; Transtornos Relacionados ao Uso de Álcool.

\section{ABSTRACT}

The aim of this study was to identify predictive risk factors for psychological distress in adolescents who live or do not live with alcoholic family members. An epidemiological study was conducted with a selection of 211 adolescents under psychological distress from a sample of 715 students from the state education network of João Pessoa, state of Paraíba, Brazil. Data were collected using three instruments: the Family CAGE; the Self-reporting Questionnaire and a structured questionnaire. The logistic regression model was used for analysis. The results evidenced that adolescents who lived with alcoholic family members whose mothers had higher education levels had lower chances of manifesting psychological illnesses $(O R=0.63)$. Among adolescents who did not live with alcoholic family members, the highest chances for manifesting illnesses were associated with the education level of their fathers $(O R=1.2)$, being female $(O R=1.8)$, and belonging to a non-nuclear family. The planning of actions/interventions that favor the effective construction of care for these individuals must take into consideration the socio-cultural singularities of the family context.

Descriptors: Psychiatric Nursing; Adolescent; Risk Factors; Alcohol-Related Disorders. 


\section{INTRODUÇÃO}

O alcoolismo na contemporaneidade apresenta-se como uma das problemáticas de grande interesse para a saúde pública, tendo em vista o crescente número de prejuízos ocasionados pelo uso excessivo de bebidas alcoólicas. Embora o consumo do álcool seja evidenciado historicamente desde relatos de séculos passados, os problemas associados ao seu consumo nem sempre foram os mesmos ou tiveram a mesma dimensão. Observa-se que mudanças no padrão de consumo ${ }^{(1-2)}$ vêm determinando sérios danos à saúde biopsicossocial da população mundial ${ }^{(3)}$.

Globalmente, o consumo nocivo de álcool provoca a cada ano cerca de 3,3 milhões de mortes (ou 5,9\% de todas as mortes), e 5,1\% da carga global de doenças é atribuível ao consumo de álcool. Em países com menor riqueza econômica, os riscos de morbidade e mortalidade são mais elevados do que nos países de renda mais alta ${ }^{(1)}$.

Dentre os principais transtornos e/ou doenças associadas ao consumo de álcool, são mencionadas as doenças neuropsiquiátricas (epilepsia, depressão, ansiedade), cirrose hepática, câncer (fígado, laringe, pâncreas), hipertensão, diabetes, suicídio, violência, síndrome alcoólica fetal (SAF) e complicações no parto pré-termo, quando do uso do álcool pela gestante ${ }^{(1)}$.

No Brasil, estudo realizado em 2012, sobre consumo de álcool, mostrou que 6,8\% da população é dependente da substância. Entre os adultos que faziam uso da bebida, $32 \%$ referiram não serem capazes de conseguir parar depois de começar a beber, $10 \%$ mencionaram que alguém se machucou em consequência do seu consumo de álcool, 9\% admitiram efeito prejudicial do álcool em seus relacionamentos familiares e conjugais, e $8 \%$ tiveram prejuízos no trabalho ${ }^{(4)}$.

Como visto, para além do impacto sobre a incidência de doenças e transtornos, os danos ocasionados pelo uso do álcool não se limitam apenas ao usuário. O consumo do álcool repercute negativamente na sua vida pessoal, profissional, financeira, social, estendendo-se aos relacionamentos familiares. A dependência química prejudica o convívio familiar atingindo todos os indivíduos próximos ao alcoolista, estabelecendo, assim, um clima de angústia, tensão, culpa e ansiedade que pode marcar o início de uma desorganização familiar ${ }^{(5)}$.

Logo, a convivência com um alcoolista na família pode contribuir para o sofrimento psíquico de todos os membros, comprometendo principalmente crianças e adolescentes que vivenciam seu processo de formação e desenvolvimento pessoal. Como estão expostos à situações corriqueiras de risco para o sofrimento mental, geralmente eles desenvolvem sintomas de depressão, ansiedade, problemas de comportamento e de aprendizagem $^{(6)}$.

Nos últimos anos, observa-se um crescimento no percentual de doenças psíquicas em adolescentes, onde $20 \%$ desses indivíduos apresentam algum tipo de sofrimento mental ${ }^{(7)}$. Quando associado ao convívio familiar com alcoolistas, verifica-se um percentual de $23 \%$ de adolescentes com sofrimento psíquico, a exemplo da depressão ${ }^{(8)}$.

Baseado em pesquisas realizadas no estado da Paraíba, que identificaram frequências de consumo de álcool entre 41 e $71 \%$ da população adulta e de adolescentes $^{(9-10)}$, bem como as implicações que seu uso excessivo pode ocasionar na saúde mental dos familiares.

O presente estudo se propõe a responder ao seguinte questionamento: quais os fatores de risco preditores para sofrimento psíquico em adolescentes que convivem ou não com familiares alcoolistas?

Portanto, objetivou-se identificar fatores de risco preditores para sofrimento psíquico em adolescentes que convivem ou não com familiares alcoolistas.

\section{MÉTODOS}

Trata-se de um estudo epidemiológico de base populacional do tipo transversal, realizado com procedimento comparativo. A população constituiu-se de 21.214 adolescentes matriculados em escolas estaduais de ensino médio em João Pessoa/PB. 
Para o cálculo amostral, admitiu-se um nível de significância de 5\%, um erro amostral de 3\% e nível de confiança de $95 \%(Z=1,96)$. Adotou-se o valor antecipado para proporção populacional (P) igual a $20 \%$. Obteve-se por meio da equação $n=P$. $(1-P) \cdot Z^{2} / e^{2}$, a amostra mínima de 662 adolescentes, adicionando-se 10\% para possíveis perdas, finalizando com amostra representativa de 715 adolescentes.

Realizou-se o processo de amostragem sistemática, a partir uma planilha eletrônica com a listagem de todos os alunos matriculados por turma em cada escola da rede estadual de ensino. Na sequência, foi feita a equação: N/n $(21.214 / 662=29)$ e o número encontrado determinou o tamanho do intervalo dado entre um entrevistado e outro, respeitando a ordem aleatória da planilha.

Os dados foram coletados no período de julho a outubro de 2011, por meio de fontes primárias (entrevistas), utilizando-se três instrumentos: CAGEfamiliar (Cutting Down, Annoyed by Criticism, Guilty $e$ Eyer-opener) - uma das ferramentas de rastreamento mais simples e confiáveis conhecidas para detectar o alcoolismo familiar; o Self-Reporting Questionnaire (SRQ20) - empregado para identificar transtornos mentais em populações sem a presença de especialistas; e um questionário estruturado para identificar as variáveis sociodemográficas e familiares.

Para este estudo, foram selecionados 211 adolescentes com sofrimento mental, classificados segundo a convivência ou não com familiares alcoolistas.

Para a análise estatística, foi utilizado o modelo de regressão logística com a proposta de avaliar os índices de sofrimento psíquico nas duas populações e identificar fatores preditivos de risco que podem contribuir para a manifestação da doença.

Para construção do modelo, foi aplicado o teste de associação de qui-quadrado entre as variáveis explicativas duas a duas, com o intuito de observar a existência de alguma associação. Como o pressuposto do modelo de regressão logística é de que essas variáveis não estejam associadas, então, as variáveis consideradas no modelo foram aquelas com $\mathrm{p}$-valor $>0,25$. Assim sendo, foi possível gerar os modelos de regressão logística múltipla, construídos a partir da introdução de todas essas variáveis e, em seguida, foram eliminadas aquelas que, em conjunto, não se apresentaram estatisticamente significativas ( $p$-valor $>0,05)$.

Para estimar os modelos logísticos, foi utilizado o software "R", versão 2.010.0, e o método consistiu na remoção sucessiva das variáveis menos significativas, permanecendo, apenas, parte das variáveis que se apresentavam significativas com nível de significância menor que $5 \%$.

Este estudo atendeu às normas que contemplam os requisitos éticos propostos pela Resolução 196/96 do Conselho Nacional de Saúde (CNS), que dispõe de normas e diretrizes regulamentadoras da pesquisa envolvendo seres humanos. O projeto foi aprovado pelo Comitê de Ética do Centro de Ciências da Saúde da Universidade Federal da Paraíba (CCS/UFPB), sob protocolo de $\mathrm{n}$ 은 066/11.

\section{RESULTADOS}

Para o grupo de adolescentes com sofrimento psíquico que conviviam com familiares alcoolistas, permaneceram como parte do modelo as variáveis: escolaridade do pai ( $p$-valor $=0,004$ ); escolaridade da mãe $(p$-valor $=0,008)$; chefe da família $(p$-valor $=0,088)$ (Tabela 1). Já para o grupo de adolescentes com sofrimento psíquico que não conviviam com familiares alcoolistas: sexo ( $p$-valor $=0,001)$; escolaridade do pai ( $p$ valor =0,026); tipo de família ( $p$-valor =0,146); chefe da família ( $p$-valor $=0,171)$; renda familiar $(p$-valor $=0,163)$ (Tabela 2).

Quanto ao grupo de adolescentes com sofrimento psíquico que conviviam com familiares alcoolistas, somente a variável chefe de família não teve associação com as outras variáveis investigadas, tornando-se assim viável referência para a construção do modelo de regressão. 
Tabela 1: Variáveis explicativas para o grupo de adolescentes com sofrimento psíquico que convivem com familiares alcoolistas. João Pessoa, PB, Brasil, 2011.

\begin{tabular}{cccc}
\hline & Escolaridade do pai & Escolaridade da mãe & Chefe da família \\
\hline Escolaridade do pai & - & $\mathbf{0 , 0 0 0 *}$ & 0,074 \\
Escolaridade da mãe & $\mathbf{0 , 0 0 0 *}$ & - & 0,736 \\
Chefe da família & 0,074 & 0,736 & - \\
\hline
\end{tabular}

$* p<0,05$ Teste qui-quadrado

Tabela 2: Variáveis explicativas do grupo de adolescentes com sofrimento psíquico que não convivem com familiares alcoolistas. João Pessoa, PB, Brasil, 2011.

\begin{tabular}{cccccc}
\hline & Sexo & Escolaridade do pai & Tipo de família & Chefe da família & Renda familiar \\
\hline Sexo & - & 0,107 & 0,311 & 0,380 & 0,676 \\
Escolaridade do pai & 0,107 & - & 0,739 & $\mathbf{0 , 0 0 2 *}$ & $\mathbf{0 , 0 0 0 *}$ \\
Tipo de família & 0,311 & 0,739 & - & 0,195 & 0,355 \\
Chefe da família & 0,380 & $\mathbf{0 , 0 0 2 *}$ & 0,195 & - & $\mathbf{0 , 0 0 0 *}$ \\
Renda familiar & 0,676 & $\mathbf{0 , 0 0 0 *}$ & 0,355 & $\mathbf{0 , 0 0 0 *}$ & - \\
\hline
\end{tabular}

* $p<0,05$ Teste qui-quadrado

Já para o grupo de adolescentes com sofrimento psíquico que não conviviam com familiares alcoolistas, foi possível verificar que as variáveis sexo e tipo de família apresentaram hipótese de associação rejeitada ( $p$-valor > $0,25)$. Quando associadas às outras variáveis, essas se constituíram em viáveis referências para a construção de um modelo de regressão logística múltipla para o não adoecimento mental do indivíduo.
Modelo de regressão logística para o sofrimento psíquico em adolescentes que conviviam com familiares alcoolistas

Na Tabela 3, estão dispostas as variáveis estatisticamente significantes ( $p$-valor $<0,05)$ para identificar o sofrimento psíquico em adolescentes que conviviam com familiares alcoolistas, seguidas de seus respectivos coeficientes, Odds Ratio e intervalos de confiança.

Tabela 3: Relação da variável familiar significativa para manifestação do sofrimento psíquico em adolescentes que convivem com familiares alcoolistas. João Pessoa, PB, Brasil, 2011.

\begin{tabular}{cccccc}
\hline \multirow{2}{*}{ Variável } & \multirow{2}{*}{ Coeficiente } & OR & \multicolumn{2}{c}{ IC (95\%) } & \multirow{2}{*}{ p-valor * } \\
& & & Inferior & Superior & 0,000 \\
Escolaridade da mãe & $-0,462$ & 0,63 & 0,49 & 0,82 & 0,000 \\
Constante & 2,147 & 8,56 & & & \\
\hline
\end{tabular}

$* p<0,05$

Neste grupo, verificou-se que apenas a variável escolaridade da mãe foi estatisticamente significativa ( $p$ valor $<0,05)$. Adolescentes, cujas mães apresentavam maior nível de escolaridade, tiveram menor chance de manifestar adoecimento psíquico $(\mathrm{OR}=0,6)$, representando um fator de proteção. Inversamente, o menor nível de escolaridade da mãe representou maior probabilidade de adoecimento psíquico.
Modelo de regressão logística para o sofrimento psíquico em adolescentes que não conviviam com familiares alcoolistas

Quanto às variáveis estatisticamente significantes ( $p$ valor $<0,05)$ para identificar o sofrimento psíquico em adolescentes que não conviviam com familiares alcoolistas, foi verificado que a variável tipo de família caracterizou-se como um fator de proteção para esse grupo. Adolescentes, cujas famílias foram classificadas como do tipo nuclear, tiveram 1,5 vezes menor chance de 
apresentar sofrimento psíquico (Tabela 4).

Tabela 4: Variáveis sociodemográficas e familiares significativas para manifestação do sofrimento psíquico em adolescentes que não convivem com familiares alcoolistas. João Pessoa, PB, Brasil, 2011.

\begin{tabular}{ccccc} 
Variável & Coeficiente & OR & \multicolumn{2}{c}{ IC (95\%) } \\
& & & Inferior & Superior \\
Sexo & 0,616 & 1,85 & 1,27 & 2,69 \\
Escolaridade do pai & 0,181 & 1,20 & 1,05 & 0,001 \\
Tipo de família & $-0,217$ & 0,80 & 0,66 & 0,008 \\
\hline
\end{tabular}

$* p<0,05$

No que diz respeito ao sexo dos adolescentes investigados, verificou-se que adolescentes do sexo feminino tiveram 1,8 vezes mais chance para o sofrimento psíquico do que adolescentes do sexo masculino. Analogamente, maior nível de escolaridade do pai aumentou em 1,20 vezes a chance para manifestação do agravo nos adolescentes.

\section{DISCUSSÃO}

O presente estudo traz informações relevantes sobre os fatores que podem funcionar como proteção ou risco para a saúde mental em um grupo de adolescentes, seja relacionando a uma situação de convivência com familiares alcoolistas ou não.

Observou-se que no grupo de adolescentes com sofrimento psíquico que conviviam com familiares alcoolistas, que o baixo nível de escolaridade materna representou um fator preditor de risco, com maior chance de manifestar o agravo.

Estudo brasileiro analisou a associação de determinantes sociodemográficos e o desenvolvimento de problemas de comportamento em escolares, verificou que crianças e adolescentes vivendo em condições sociais precárias, cujos pais tinham baixa escolaridade, a presença de problemas de comportamento foi mais frequente. Logo, a convivência em família com desvantagem educacional influencia negativamente a saúde mental dos filhos ${ }^{(11)}$.

As pessoas que possuem maior grau de escolaridade têm melhores colocações no mercado de trabalho, possuem maior renda familiar, têm acesso às melhores condições de vida e, consequentemente, proporcionam condições satisfatórias aos seus familiares como educação, lazer, saúde, moradia, ou seja, são supridas as necessidades de sobrevivência ${ }^{(12)}$, características que, na maioria das vezes, funcionam como fator de proteção para preservação da saúde mental do indivíduo.

Considerando que o adolescente é influenciado diretamente por sua família na sua formação biopsicossocial, quando se verifica nesse núcleo pessoas que fazem uso abusivo de bebidas alcoólicas, geralmente, o cotidiano é marcado por relações afetivas fragilizadas, com distanciamento entre seus membros em consequência de conflitos e crises existenciais frequentes. Portanto, o adolescente apresenta um nível elevado de ansiedade, ou mesmo, um sentimento de impotência diante da situação vivenciada, resultando, muitas vezes, em sofrimento físico e psíquico ${ }^{(13)}$.

Mesmo considerando as evidências de que em famílias de alcoolistas os membros são mais vulneráveis para a manifestação do sofrimento psíquico, quando se identifica neste estudo que a precária educação materna influencia a manifestação do agravo, pressupõe-se que mães com maior escolaridade têm disponibilidade ampliada para dar suporte emocional aos filhos. $\mathrm{O}$ acesso à informação e aos meios de comunicação favorece o desenvolvimento de habilidades para que essas mães reconheçam alterações comportamentais significativas, aumentem as oportunidades de diálogo e suporte para a identificação das necessidades e demandas por eles apresentadas $^{(14-15)}$. 
Para os adolescentes com sofrimento psíquico que não conviviam com familiares alcoolistas, o modelo logístico gerado mostrou que o tipo de família apresentou-se como um fator preditor de risco para este grupo. Observou-se que indivíduos com famílias de modelos diferentes do tipo nuclear tiveram mais chance de apresentar sofrimento psíquico.

A dinâmica familiar é algo imprescindível para a compreensão do ser humano enquanto elemento social, pois é no meio familiar que ele empreende sua constituição individual, desenvolvimento psicológico, personalidade e a organização de sua identidade ${ }^{(16)}$. Constitui-se, assim, como uma das necessidades do indivíduo, visto que nela ocorrem as primeiras trocas afetivas emocionais, na qual se vivenciam momentos de alegria, de tristeza, mas é no seu interior que o sujeito aprende a se definir como diferente e a enfrentar seus conflitos durante o processo de crescimento pessoal ${ }^{(13-14)}$.

Logo, infere-se que uma coesão familiar adequada, uma boa relação com os pais e convivência em famílias nucleares funcionam como fatores de proteção para o adoecimento mental dos seus membros ${ }^{(17)}$. A dissociação dos laços familiares, por vezes, gera consequências marcantes na vida das crianças e adolescentes, podendo levar a diversos distúrbios de comportamento ${ }^{(13)}$.

Outro resultado apresentado para este grupo está relacionado à variável sexo que se apresentou como fator preditor de risco, uma vez que adolescentes do sexo feminino tiveram aumentadas as chances para manifestar o sofrimento psíquico. Segundo autores ${ }^{(18-19)}$, essa predisposição está relacionada às pressões impostas devido à expansão de seus papéis, ao excesso de trabalho, à discriminação de gênero, à violência doméstica e sexual.

É importante perceber que as desigualdades de gênero podem privilegiar o sexo masculino e afetar a autoestima feminina, devido aos valores sociais machistas ainda predominantes na sociedade atual; assim, introjeta-se que a dominação masculina tem as condições favoráveis em toda a estrutura social ${ }^{(20)}$.
Diante dessas constatações, considerar que a saúde mental está baseada tanto no bem-estar pessoal como nos contextos sociais é importante para a proposição de políticas públicas para jovens e adolescentes, reconhecendo que estes aspectos são fundamentais para um desenvolvimento saudável ${ }^{(21)}$.

Ainda no grupo de adolescentes que não conviviam com familiares alcoolistas, constatou-se que quanto maior a escolaridade do pai, maior a chance para manifestação do sofrimento psíquico no grupo. Dentre os motivos, destaca-se uma jornada de trabalho maior e exaustiva, reduzindo, consequentemente, a presença da figura paterna no lar e sua participação na família.

O pai tem ocupado novo espaço no seio familiar, não se configura apenas como provedor ou autoridade da família, mas tem compartilhado com a mãe atividades cotidianas dos filhos que vão desde a educação até brincadeiras e momentos de afetuosidade. Um maior envolvimento dele nas atividades referentes à criança contribui para uma boa formação psicossocial ${ }^{(22)}$.

A relação entre pais e filhos é fruto de um conjunto de expectativas compartilhadas, e que o envolvimento entre esses indivíduos é importante para o seu desenvolvimento físico e mental. De tal modo, destaca-se a necessidade de cuidar do adolescente de modo ampliado, para que ele possa estar inserido em uma rede de atenção social capaz de suprir as necessidades do ser em desenvolvimento.

Ressalta-se, ainda, a importância da equipe de profissionais de saúde na rede social de atenção à família, e a posição estratégica de que a enfermagem deve ter no cuidado à saúde, desempenhando uma atenção mais próxima e humanizadora, priorizando ações de prevenção e identificação dos fatores de risco, em razão de suas implicações para o adolescente e para o meio social ${ }^{(23)}$.

Os resultados obtidos indicaram as variáveis que apresentaram maior influência para manifestação do adoecimento psíquico em adolescentes, e que, portanto, devem ser priorizadas por meio de intervenções específicas. Por se tratar de um desafio da saúde pública 
do Brasil, o uso de álcool e de outras drogas psicoativas tem se tornado um tema constante e preocupante para famílias, profissionais de saúde, da educação e autoridades governamentais, visto o seu avanço crescente na população brasileira ${ }^{(24)}$.

A construção de propostas de ações preventivas deve ter a participação de todos os envolvidos no contexto social do público a ser atingido, respeitando a expressão da singularidade e valorizando a necessidade individual.

O tema referente às drogas está a cada dia mais presente na vida da população brasileira, principalmente de crianças e adolescentes. Dessa feita, a escola e todos os que dela fazem parte são cada vez mais cobrados para a abordagem desta questão de forma mais significativa, ao tratá-la preventivamente com os alunos e de modo mais efetivo com aqueles que necessitam atenção especial, por sua vulnerabilidade.

A família que convive com um membro alcoolista deveria caracterizar-se como uma prioridade no contexto das políticas de saúde, especialmente com foco direcionado à saúde mental, tendo em vista que todos os membros da família são vulneráveis a agravos ao ter que compartilhar a situação do alcoolismo vivenciada por um familiar ${ }^{(13,16)}$.

\section{CONCLUSÃO}

Esta investigação permitiu identificar fatores preditores de risco para o sofrimento psíquico relacionado ao alcoolismo familiar em adolescente. Em famílias de alcoolistas, os membros são mais vulneráveis à manifestação do sofrimento psíquico. Por sua vez, tal adoecimento psíquico ocorre mais frequentemente entre os adolescentes, quando se identifica no núcleo familiar que a mãe apresenta menor grau de escolaridade.

Constatou-se, ainda, que o sofrimento psíquico em grupos de adolescentes que não convivem com familiares alcoolistas acontece em maior probabilidade quando esses são do sexo feminino, os pais têm maior escolaridade e as famílias possuem modelo não convencional, ou seja, tradicional nuclear.

Considerando as constantes mudanças e evolução em todas as áreas do conhecimento, torna-se necessário o desenvolvimento de pesquisas científicas para incentivar a adoção de práticas preventivas de forma que todos se sintam corresponsáveis para a formação de uma consciência preventiva quanto ao uso abusivo de álcool e outras drogas em todos os ambientes sociais.

Há que se considerar que as atividades de promoção à saúde mental caracterizam-se como de grande relevância no meio social, tendo em vista que o problema do consumo de álcool e outras substâncias psicoativas tem se constituído uma ameaça à estabilidade dos valores familiares, sociais, culturais, econômicos e políticos no Brasil.

Os fatores de risco identificados no estudo são marcadores de grande magnitude social e podem subsidiar o planejamento de ações/intervenções que favoreçam a construção efetiva de cuidados a esses indivíduos. Enfatiza-se, contudo, que o problema deve ser analisado sob enfoques mais amplos que considerem as singularidades socioculturais do contexto familiar e as múltiplas determinações existentes.

\section{REFERÊNCIAS}

1. World Health Organization. Global status report on alcohol and health 2014. Geneva: World Health Organization, Department of Mental Health and Substance Abuse 2014. [ Acesso em: 30 jan 2015]. Disponível em:

http://apps.who.int/iris/bitstream/10665/112736/1/9789240692763_ eng.pdf

2. Alves VS, Lima, Oliveira IMS. Atenção à saúde de usuários de álcool e outras drogas no brasil: convergência entre a saúde pública e os direitos humanos. R Disan [internet]. 2013[acesso em: 20 Dez 2014];13 (3): 9-32. Disponível em: http://dx.doi.org/10.11606/issn.2316-9044.v13i3p9-32

3. Oliveira GF, Luchesi LB. O discurso sobre álcool na Revista Brasileira de Enfermagem: Rev Lat Am Enfermagem [internet]. 2010[ acesso em: 22 dez 2014]; 18 Spec: 626-33. Disponível em: http://www.scielo.br/scielo.php?pid=S0104-11692010000700020\&script=sci_arttext 4. Instituto Nacional Ciência e Tecnologia para Políticas Públicas de Álcool e Outras Drogas (INPAD). II Levantamento Nacional de Álcool e Drogas 2012 (LENAD) [internet]. Ronaldo Laranjeira (Supervisão)[et al.]. São Paulo: UNIFESP; 2014. [acesso em: 28 dez 2014]. Disponível 
em: http://inpad.org.br/wp-content/uploads/2014/03/Lenad-II-Relat\%C3\%B3rio.pdf.

5. Son JY, Choi YJ. The effect of an anger management program for family members of patients with alcohol use disorders. Arch Psychiatr Nurs. 2010;24(1):38-45.

6. Souza J, Carvalho AMP. Repercussões do ambiente familiar alcoolista para o desenvolvimento da criança: relato de caso. Pediatr Mod.[ internet] 2010[acesso em: 10 jan 2014];46(3):114-9. Disponível em:

http://www.moreirajr.com.br/revistas.asp?fase=r003\&id materia $=4357$

7. World Health Organization. Child and adolescent Mental Health Polices and plans. Mental health policy and services guidance package. WHO;2005[citado 2014 dez.18].Disponível em:

http://www.who.int/mental_health/policy/Childado mh module.pdf 8. Barrientos VA, Mendoza SHF, Sainz LV, Pérez CH, Gil Al, Soler EH. Depresión y tipología familiar en un grupo de adolescentes mexicanos. Arch Med Fam [internet]. 2010[ acesso em: 23 jan 2015];12(3):69-76. Disponível em: http://www.medigraphic.com/pdfs/medfam/amf2010/amf103b.pdf

9. Cerqueira GS, Lucena CT, Gomes ATM, Freitas APF, Rocha NFM, Mariz SR.Consumo de álcool entre estudantes de uma escola pública da cidade de Cajazeiras, PB. Revista electronica salud mental, alcohol y drogas [internet].2011[acesso em:03 fev 2015];7(1):18-24.Disponível em: http://www.revistas.usp.br/smad/article/view/38735

10. Melo JRF, Maciel JRSC, Oliveira RCC, Silva AO. Implicações do uso do álcool na comunidade indigena Potiguara. Physis [internet], 2011 [acesso em: 12 jan 2015]; 21(1):319-33. Disponível em: http://www.scielo.br/scielo.php?pid=S0103-73312011000100019\&script=sci_arttext

11. Assis SG, Avanci JQ, Vasconcellos R, Oliveira C. Desigualdades socioeconômicas e saúde mental infantil. Rev Saúde Pública [internet],2009[acesso em: 20 jan 2015];43(Supl.1):92-100.Disponível em: http://www.scielo.br/sci-

elo.php?pid=\$003489102009000800014\&script=sci_arttext

12. Instituto Brasileiro de Geografia e Estatística [internet]. Brasília: Ministério do Planejamento, Orçamento e Gestão (BR) [acesso em: 23 Dez 2014].Indicadores sociais do censo populacional de 2010. Disponível em: http://www.ibge.gov.br/home/

13. Sena ELS, Boery RNSO, Carvalho PAL, Reis HFT, Marques AMN . Alcoolismo no contexto familiar: um olhar fenomenológico. Texto Contexto Enferm [internet]. 2011[acesso em: 05 jan 2015]; 20:310-18. Disponível em: http://www.scielo.br/scielo.php?pid=S0104-

07072011000200013\&script=sci_arttext

14. Silva DAS, Pereira IMM, Oliveira ACC. Impacto da escolaridade materna e paterna na perceção da imagem corporal em acadêmicos de Educação Física. Motricidade.[internet]2012 [acesso em 10 jan 2015]; 8(2):22-31. Disponível em : http://revistas.rcaap.pt/motricidade/article/viewFile/709/579

15.Crivelatti MMB, Durman SL, Hofstatter M. Sofrimento psíquico na adolescência. Texto Contexto Enferm [internet]. 2006 [acesso em 2011 nov.12]; 15 (esp): 64-70. Disponível em: http://www.scielo.br/scielo.php?script=sci_arttext\&pid=S0104-07072006000500007 16.Andrade FB, Bezerra AIC, Pontes ALF, Ferreira Filha MO, Vianna RPT , Dias MD, et al. Saúde Mental na Atenção Básica: um estudo epidemiológico baseado no enfoque de risco. Rev Bras Enferm [internet]. 2009 [acesso em 03 jan 2015]; 62:675-80. Disponível em:

http://www.scielo.br/scielo.php?script=sci_arttext\&pid=S003471672009000500004

17.Galvis YT, Vanegas MAS, Velez LPM, Cossio GIG, Villegas GH, Valencia M L. Segundo Estudio de Salud Mental del Adolecente. Medellín: Secretaría de Salud de Medellín - F. Medicina, Universidad CES; 2010.165p. [acesso em 02 jan 2015 ] Disponível em:

http://onsm.ces.edu.co/uploads/files/11201434_SEGUNDO-ESTUDIODE-SALUD-MENTAL-DEL-ADOLESCENTE-MEDELLIN---2009.pdf
18. Pinho PS, Araújo TM. Associação entre sobrecarga doméstica e transtornos mentais comuns em mulheres. Rev Bras Epidemiol. 2012; 15(3):560-72.

19. Albuquerque JBC, César ESR, Silva VCL, Espínola LL, Azevedo EB, Ferreira Filha MO. Violência doméstica: características sociodemográficas de mulheres cadastradas em uma Unidade de Saúde da Família. Rev Eletr Enf [Internet]. 2013 [acesso em:02 jan 2015] 15(2):382-90. Disponível em:

http://dx.doi.org/10.5216/ree.v15i2.18941

20. Franzoi NM, Fonseca RMGS, Guedes RN. Violência de gênero: concepções de profissionais das equipes de saúde da família. Rev. Lat Am Enfermagem[internet]. 2011 [acesso em: 03 jan 2015];19(3):[09 telas].Disponível em: http://www.scielo.br/sci-

elo.php?pid=S010411692011000300019\&script=sci abstract\&tlng=pt 21. Fukuda CC, Garcia KA, Amparo DM. Concepções de saúde mental a partir da análise do desenho de Adolescentes. Estudos de Psicologia[internet]. 2012[ acesso em: 27 dez 2014]; 17(2):207-214. Disponível em: http://www.scielo.br/pdf/epsic/v17n2/03.pdf

22. Benczik EBP. A importância da figura paterna para o desenvolvimento infantil. Rev. Psicopedag.[ internet]. 2011[acesso em: $01 \mathrm{fev}$ 2015];28(85):67-75. Disponível em: http://pepsic.bvsalud.org/scielo.php?script=sci_arttext\&pid=S0103-84862011000100007

23. Silva SED, Padilha MI. História de vida e o alcoolismo: representações sociais de adolescentes. reme Rev Min Enferm [internet]. 2011[ acesso em: 19 dez 2014];15(1):70-78. Disponível em: http://www.revenf.bvs.br/scielo.php?script=sci_arttext\&pid=S141527622011000100010\&lng=es\&nrm=iso\&tlng=pt

24. Silva SED, Padilha MI , Araújo JS. A interação do adolescente com o familiar alcoolista e sua influência para adicção do alcoolismo. Rev enferm UFPE [ internet].2014 [acesso em: 06 jan 2015]; 8(1):59-69.Disponível em: http://www.revista.ufpe.br/revistaenfermagem/index.php/revista/article/view/5754.

Recebido: 31/05/2013.

Aceito: 09/12/2014.

Publicado: 30/06/2015. 